\title{
Comparison of the Internal Energy Deposition of Venturi-Assisted Electrospray Ionization and a Venturi-Assisted Array of Micromachined UltraSonic Electrosprays (AMUSE)
}

\author{
Christina Y. Hampton, ${ }^{a}$ Catherine J. Silvestri, ${ }^{a}$ Thomas P. Forbes, ${ }^{\mathrm{b}}$ \\ Mark J. Varady, ${ }^{\mathrm{b}}$ J. Mark Meacham, ${ }^{\mathrm{b}}$ Andrei G. Fedorov, ${ }^{\mathrm{b}, \mathrm{c}}$ \\ F. Levent Degertekin, ${ }^{b}$ and Facundo M. Fernández ${ }^{a}$ \\ a School of Chemistry and Biochemistry, Georgia Institute of Technology, Atlanta, Georgia, USA \\ ${ }^{b}$ George W. Woodruff School of Mechanical Engineering, Georgia Institute of Technology, Atlanta, \\ Georgia, USA \\ ' Parker H. Petit Institute of Bioengineering and Bioscience, Georgia Institute of Technology, Atlanta, \\ Georgia, USA
}

The internal energy deposition of a Venturi-assisted array of micromachined ultrasonic electrosprays (AMUSE), with and without the application of a DC charging potential, is compared with equivalent experiments for Venturi-assisted electrospray ionization (ESI) using the "survival yield" method on a series of para-substituted benzylpyridinium salts. Under conditions previously shown to provide maximum ion yields for standard compounds, the observed mean internal energies were nearly identical (1.93-2.01 eV). Operation of AMUSE without nitrogen flow to sustain the air amplifier focusing effect generated energetically colder ions with mean internal energies that were up to $39 \%$ lower than those for ESI. A balance between improved ion transfer, adequate desolvation, and favorable ion energetics was achieved by selection of optimum operational ranges for the parameters that most strongly influence the ion population: the air amplifier gas flow rate and API capillary temperature. Examination of the energy landscapes obtained for combinations of these parameters showed that a low internal energy region $(\leq 1.0 \mathrm{eV})$ was present at nitrogen flow rates between 2 and $4 \mathrm{~L} \mathrm{~min}{ }^{-1}$ and capillary temperatures up to $250^{\circ} \mathrm{C}$ using ESI ( $9 \%$ of all parameter combinations tested). Using AMUSE, this region was present at nitrogen flow rates up to $2.5 \mathrm{~L} \mathrm{~min}^{-1}$ and all capillary temperatures $(13 \%$ of combinations tested). The signal-to-noise $(\mathrm{S} / \mathrm{N})$ ratio of the intact $p$-methylbenzylpyridinium ion obtained from a $5 \mu \mathrm{M}$ mixture of thermometer compounds using AMUSE at the extremes of the studied temperature range was at least fivefold higher than that of ESI, demonstrating the potential of AMUSE ionization as a soft method for the characterization of labile species by mass spectrometry. (J Am Soc Mass Spectrom 2008, 19, 1320-1329) (C) 2008 American Society for Mass Spectrometry

$\mathrm{T}$ The generation of low internal energy ions is important for the study of fragile organic compounds and noncovalent biological species such as protein complexes. The introduction of matrixassisted laser desorption ionization (MALDI) $[1,2]$ and electrospray ionization (ESI) $[3,4]$ in the late 1980s accelerated the growth of bioanalytical mass spectrometry (MS) by enabling the routine analysis of large biological molecules with minimum fragmentation. Despite the widespread appeal of these ionization tech-

Address reprint requests to Professor Facundo M. Fernández, 901 Atlantic Dr. NW, Atlanta, GA 30332-0400. E-mail: facundo.fernandez@chemistry.gatech. edu niques, there has been considerable activity toward the development of even "softer" methods, including atmospheric pressure-MALDI [5], sonic spray ionization (SSI) [6], electrosonic spray ionization (ESSI) [7], and cold spray ionization (CSI) [8], with the intent of exploring improved ways for detecting biological species such as peptides [9], proteins [10,11], noncovalent complexes [12], and nucleic acids [13].

The Array of Micromachined UltraSonic Electrosprays (AMUSE) was invented by Fedorov and Degertekin for high throughput, multiplexed MS [14] and was first demonstrated on an MS system jointly by the Fedorov, Degertekin, and Fernández groups [15]. In the AMUSE, the processes of droplet formation and DC droplet charging are decoupled. The application of a radio 
frequency (RF) signal to a piezoelectric transducer at a resonant frequency of the sample-filled cavity generates a standing ultrasonic wave within the sealed analyte reservoir and forms a pressure gradient at the micronsized orifices of the array, thus enabling efficient ejection of multiple parallel droplet streams [16]. Droplet charging is most commonly performed by directly applying a DC charging potential to an electrode placed within the analyte solution reservoir [15]. There are several intrinsic advantages of this fundamentally different ionization method. First, unlike conventional ESI - which requires the use of high DC potentials (1-5 $\mathrm{kV}$ ) to both form and charge droplets-the AMUSE provides sensitive ionization of biomolecules with only several hundred volts [17]. The inclusion of organic solvents to reduce the surface tension of the sprayed solution in ESI analysis is beneficial for facilitating Taylor cone formation but may perturb the native conformation of the solutes [18]. The AMUSE does not rely on Taylor cone formation; thus, ionization is possible from completely aqueous solutions, making it well suited for the analysis of biological systems. Finally, the array format of the AMUSE is ideally suited for multiplexing, as recently demonstrated by Forbes et al. [19].

One challenge commonly faced during atmospheric pressure ion generation is the low ion transfer efficiency within the spectrometer's atmospheric pressure interface (API) [20]. To mitigate this difficulty, several methods have been proposed including air amplifiers (also known as Venturi devices) [21, 22], flared inlet capillaries [23, 24], flared inlet capillaries coupled to air amplifiers [25], and ion funnels [26, 27]. In previous work, we incorporated an air amplifier into the AMUSE setup to improve ion yields by increasing the linear velocity of the droplet streams ejected by the device [17]. Air amplifiers are devices that rely on the Venturi and Coanda effects to focus a charged droplet cloud toward the mass spectrometer inlet [28]. Voltage- and heatassisted operation of these air amplifiers has been reported and it has been suggested that these devices provide enhanced droplet desolvation [17, 29]. Previously, we reported that the coupling of an air amplifier to the AMUSE resulted in a tenfold gain in signal-tonoise $(\mathrm{S} / \mathrm{N})$ ratio of $10 \mu \mathrm{M}$ reserpine accompanied by a $77 \%$ reduction in relative standard deviation (\%RSD) of the signal. The use of heated nitrogen $\left(\mathrm{N}_{2}\right)$ flowing through the air amplifier provided an additional 18-fold improvement in signal intensity and a 4-fold gain in $\mathrm{S} / \mathrm{N}$ ratio for an aqueous $4 \mu \mathrm{M}$ cytochrome $\mathrm{C}$ solution. Comparison of the charge-state distributions of cytochrome C obtained using Venturi-assisted AMUSE ionization, conventional ESI, and nanoelectrospray ionization showed a slight shift toward lower charge states for AMUSE spectra [17]. Despite the analytical advantages observed for AMUSE coupled to an air amplifier, its intrinsic internal energy deposition, including the contributions from the air amplifier, were unknown to date. In addition, the internal energy of ions produced by Venturi-assisted ESI was also unknown.
In this work, we provide a comparison of the internal energy deposition of Venturi-assisted AMUSE ionization and Venturi-assisted ESI. The influence of the parameters that most strongly affect desolvation-the flow rate of ambient $\mathrm{N}_{2}$ through the air amplifier and the API capillary inlet temperature-are investigated to determine their effect on internal energy deposition. Our results demonstrate that AMUSE ionization is softer than ESI at the assayed liquid flow rate using low air amplifier $\mathrm{N}_{2}$ flow rates and that the internal energy deposition by both ion sources responds differently to combinations of various experimental parameters.

\section{Experimental}

\section{Synthesis and Preparation of Para-Substituted Benzylpyridinium Salts}

Pyridine, nitromethane, anhydrous diethyl ether, anhydrous ethanol, and the para-substituted benzyl halide starting reagents were purchased from Sigma-Aldrich (St. Louis, MO, USA) and used without further purification. All compounds except the methoxy-substituted salt $\left(\mathrm{OCH}_{3}\right.$; Arkat USA, Inc., Gainesville, FL, USA) were synthesized by condensation of the para-substituted benzyl halide with pyridine followed by recrystallization from diethyl ether using the method by Katritzky et al. [30], resulting in para-substituted benzylpyridinium salts with tert-butyl- $(\mathrm{tBu})$, methyl- $\left(\mathrm{CH}_{3}\right)$, fluoro- $(\mathrm{F})$, chloro- $(\mathrm{Cl})$, cyano- $(\mathrm{CN})$, and nitro- $\left(\mathrm{NO}_{2}\right)$ substituents. Following mass spectral analysis to assess the purity of the synthesized compounds, all salts were stored in a $-80{ }^{\circ} \mathrm{C}$ freezer until needed. For all experiments, an equimolar mixture (5 $\mu \mathrm{M}$ each) was freshly prepared in either a 50\% methanol solution (HPLC grade, Sigma-Aldrich) or nanopure water (Barnstead International, Dubuque, IA, USA) before analysis and delivered to the ion source under investigation at a flow rate of $20 \mu \mathrm{L} \mathrm{min}^{-1}$, the minimum flow rate possible for this particular AMUSE device, using a liquid handling pump (Valco Instruments Co., VICI M6, Houston, TX, USA). No organic acids were used because the analytes were precharged.

\section{AMUSE Fabrication, Assembly, and Operation}

Fabrication of the $20 \times 20$ array of $5-\mu \mathrm{m}$ nozzles in silicon [31] and assembly of the AMUSE ion source [17] have been described in detail elsewhere. Briefly, a gold-coated piezoelectric transducer (American Piezo Ceramics Inc., Mackeyville, PA, USA) was separated from the silicon ejector array by a series of silicone spacers (McMaster-Carr, Atlanta, GA, USA) that were used to define the volume of the analyte reservoir. The entire assembly was clamped using spring-loaded clips and the sample was introduced to the AMUSE through PEEK tubing (Upchurch, Oak Harbor, WA, USA), which extended from the liquid handling pump to the reservoir. The reservoir was primed with fresh solution 
before all experiments. An RF amplifier (T\&C Power Conversion Inc., Rochester, NY, USA) was used to generate a $910-\mathrm{kHz} \mathrm{RF}$ sine wave as measured on an oscilloscope (Tektronix, Beaverton, OR, USA). The amplified RF signal was applied to the metal-coated, exterior surface of the piezoelectric transducer to bring about acoustic ejection of liquid droplets with an average diameter of 5-7 $\mu \mathrm{m}$ [31]. Experiments were performed in two modes: (1) without an applied DC potential ("RF-only AMUSE") and (2) with a $100 \mathrm{~V}_{\mathrm{DC}}$ potential (Stanford Research Systems Inc., Sunnyvale, CA, USA) directly applied to the analyte reservoir via a connection to the electrode-coated, interior surface of the piezoelectric crystal ("DC-AMUSE").

\section{Venturi-Assisted ESI Setup}

The needle assembly of a commercial ESI source (ThermoFinnigan, Waltham, MA, USA) fitted with an $83 \mu \mathrm{m}$ i.d. spray capillary (Small Parts, Miramar, FL, USA) was removed from the source housing and mounted to a post using a clamp. The needle was centered with respect to an air amplifier (HMC Brauer Co., Milton Keynes, UK) and positioned so that the needle tip to air amplifier distance was equal to that used for the AMUSE. A 4-kV DC electric potential was applied to the needle using the built-in power supply of a quadrupolar ion trap (QiT) mass spectrometer (LCQ Deca XP+, ThermoFinnigan) to produce a stable electrospray.

\section{Air Amplifier Setup and Operation}

Before mass spectrometric analysis, the ion source under study was placed $4 \mathrm{~mm}$ in front of the inlet of the air amplifier, the minimum distance possible using the current AMUSE assembly. Unless otherwise specified, the air amplifier was positioned so that its outlet was flush relative to the API capillary inlet, resulting in a total distance of $77 \mathrm{~mm}$ between the point of droplet emission and the inlet orifice as shown in Figure 1. A differential pressure flow controller (Bel-Art, Pequannock, NJ, USA) was used to control the flow rate $(0-10.6$ $\mathrm{L} \min ^{-1}$ ) of industrial-grade $\mathrm{N}_{2}$ gas (130 psi; Airgas, Radnor, PA, USA) used to provide suction of the

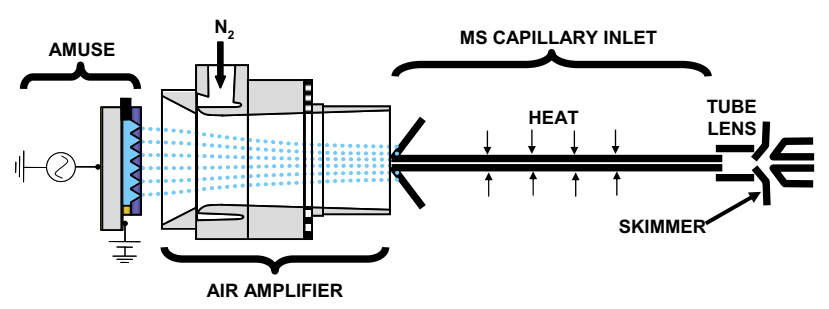

Figure 1. Setup of the Venturi-assisted AMUSE experiment and relevant components of the mass spectrometer's API. Venturiassisted ESI experiments used an identical setup whereby the AMUSE was replaced by an ESI needle positioned so that the exit of the source was about $4 \mathrm{~mm}$ from the air amplifier inlet (drawing not to scale). droplet streams through the low-pressure region in the center of the air amplifier. Supplementary Table S-1, which can be found in the electronic version of this article, provides the gas pressures that correspond to the experimental flow rates observed if gas flow was controlled solely by a pressure regulator. Droplet size distributions were measured in triplicate with a laser diffraction particle-size analyzer equipped with a 63-mm focal length optical collection system (Model 2600C, Malvern, Worcestershire, UK). The correct performance of this instrument was verified by interrogating a calibrated graticule $(46.5-\mu \mathrm{m}$ nominal particle size), and particle-size measurements were found to be accurate within $4 \%$. All particle size measurements were performed perpendicular to the ejection axis, with the laser measurement area placed immediately at the exit of the air amplifier. The area over which time-averaged particle-size measurements were obtained is approximately cylindrical in shape, and $22 \mathrm{~mm}$ long $\times 7 \mathrm{~mm}$ diameter.

\section{Mass Spectrometry}

A commercial ESI source (ThermoFinnigan) was interfaced to the QiT and used to optimize the ion optics and mass analyzer settings to obtain maximum signal intensity for the base peak $(\mathrm{m} / \mathrm{z} 226$; precursor ion of the p-tert-butyl substituted compound) using the autotune function. These settings were used for all subsequent experiments. For all experiments performed, the inlet capillary voltage was set to $-30 \mathrm{~V}$, whereas the capillary temperature was varied between 230 and $300^{\circ} \mathrm{C}$ and the tube lens potential offset (TL) was varied from 0 to $100 \mathrm{~V}$ to modify the desolvation/declustering conditions. The automatic gain control was kept on and set to a maximum of $5 \times 10^{7}$ ions, with a maximum trap time of $100 \mathrm{~ms}$ ( 3 microscans) for all experiments. Mass spectra were acquired for $1 \mathrm{~min}$.

\section{Survival Yield Method}

Only a brief description of the survival yield method is provided here. This method correlates the fragmentation extent of a thermometer molecule with the internal energy deposited on it during the ionization process [32]. This method is predicated on two assumptions: (1) all ions having the same degrees of freedom (DOF) have identical internal energy distributions and (2) ions do not dissociate until their internal energy surpasses their intrinsic dissociation threshold. The survival yield (SY) of a thermometer ion (eq 1) can be determined experimentally by measuring the fraction of ions below (product ions) and above (precursor ions) the dissociation threshold of a given molecule, according to

$$
S Y_{\text {Exp }}=\frac{\text { Int }_{\text {Precursor }}}{\text { Int }_{\text {Precursor }}+\text { Int }_{\text {Product }}}
$$




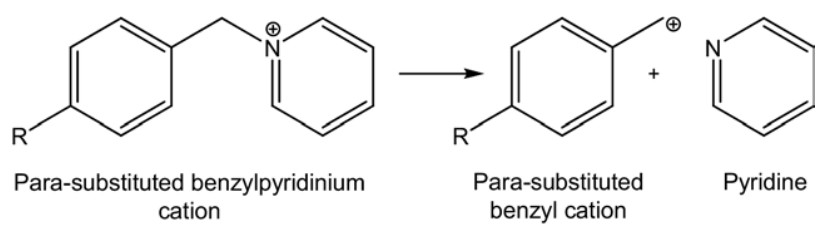

Scheme 1

This is theoretically equivalent to the value of the integral below the internal energy distribution curve $[P(E)]$ evaluated from $E=0$ to $E_{0}$, the dissociation energy of the molecule. Collette and De Pauw introduced the use of substituted benzylpyridinium salts as probe ions because they undergo a simple cleavage reaction at known dissociation energies to form a benzyl cation and pyridine (Scheme 1) [33].

By using a series of para-substituted benzylpyridinium salts that have a similar number of DOF but differing dissociation energies [34], the simultaneous measurement of the survival yield of each molecule under controlled experimental conditions is obtained. The survival yields of these compounds plotted against their respective dissociation energies can then be fitted to a sigmoidal function with the general form described in the following equation, which is differentiated to yield the internal energy distribution:

$$
\mathrm{SY}_{\mathrm{Fitted}}=\frac{\mathrm{a}}{1+\mathrm{e}^{-\left(\frac{\text { Energy }-\mathrm{x}_{0}}{\mathrm{~b}}\right)}}=\int_{0}^{\infty} \mathrm{P}(\mathrm{E}) \mathrm{dE}
$$

The parameters $a, b$, and $x_{0}$ in eq 2 are variables fitted to the experimental data. Finally, the mean internal energy $(\langle\mathrm{E}\rangle)$ of the ions is calculated by finding the centroid of the internal energy distribution, according to

$$
\langle E\rangle=\frac{\int_{0}^{\infty} E \cdot P(E) d E}{\int_{0}^{\infty} P(E) d E}
$$

The observed mean internal energy deposited not only depends on the intrinsic dissociation rate constant and activation energy, but is also strongly influenced by the timescale of the mass spectrometric experiment, manifested as the excess energy ("kinetic shift") required to observe dissociation products. For absolute measurements, correction for this kinetic shift is necessary, but can be a challenging task because it requires knowledge of the ions' residence times as they are transported and analyzed within the different stages of the mass spectrometer. However, a comparison of the relative changes in the internal energy distribution as a result of using different ionization techniques can be accurately obtained if the residence time remains consistent throughout all experiments or/and if the survival yields of the ions used in calculating internal energy distributions do not vary significantly within the experimental timescale. Experiments performed at trap- ping times ranging from 2 to $100 \mathrm{~ms}$ showed that the survival yields remained constant within experimental error for all thermometer molecules, except the tBu compound, resulting in mean internal energies that varied by $<1 \%$ for each ionization technique (Supplementary Figure S-1). This indicated that, even if fluctuations in ion source intensity caused the trapping time to be automatically adjusted by the instrument's software, the $P(E)$ curves would not be significantly affected. The survival yield values for the tBu thermometer compound were not used in the internal energy calculations.

\section{Data Analysis}

Each mass spectrum was averaged for 1 min using the built-in mass spectrometer software (Xcalibur 2.0, ThermoFinnigan) and exported into Excel (Microsoft, Auburn, WA, USA). A macro was used to identify the peak maxima for each of the ions of interest and to calculate the survival yields of each species that were then exported into SigmaPlot (Systat Software Inc., San Jose, CA, USA) and plotted against their respective appearance energies $\left(1.508,1.767,1.867,1.899,2.097\right.$, and $2.352 \mathrm{eV}$ for $\mathrm{OCH}_{3}$, $\mathrm{CH}_{3}, \mathrm{~F}, \mathrm{Cl}, \mathrm{CN}$, and $\mathrm{NO}_{2}$ p-substituents, respectively [34]). A series of SigmaPlot macros were used to fit the experimental survival yields to a sigmoidal curve and to calculate the residual error of the fit, internal energy distribution, mean internal energy, and the full width halfmaximum (FWHM) of the distribution. Two alternate methods of fitting the experimental survival yield curves were applied to the data as described in detail by Gabelica et al. [34], and the simplest model providing the highest correlation coefficient was chosen.

\section{Results and Discussion}

\section{Internal Energy Deposition Under Optimized Desolvation Conditions}

In our prior studies of Venturi-assisted AMUSE ionization, it was found that maximum ion yields for a variety of test compounds were obtained using an API capillary temperature of $300{ }^{\circ} \mathrm{C}$ and an air amplifier $\mathrm{N}_{2}$ flow rate of $8.76 \mathrm{~L} \mathrm{~min}^{-1}$ [17]. The internal energy distributions observed for ESI, RF-only AMUSE, and DC-AMUSE ionization of a 5- $\mu \mathrm{M}$ mixture of thermometer molecules prepared in $50 \%$ methanol/water, with and without the use of ambient $\mathrm{N}_{2}$ flowing through the air amplifier, are shown in Figure 2a and b. Corresponding mass spectra are shown in Figure S-2. Examination of the ESI spectra (Figure S-2a and b) showed a relatively small difference in mean internal energy for operation with and without $\mathrm{N}_{2}$ flowing through the air amplifier (Figure 2a versus $2 b$ ), and a clear improvement in signal intensity. This effect was not as obvious for the AMUSE spectra (Figure S-2c-f) because there was also a marked increase in the mean internal energy (Figure 2a versus $2 b$ ), which caused a corresponding decrease in signal inten- 

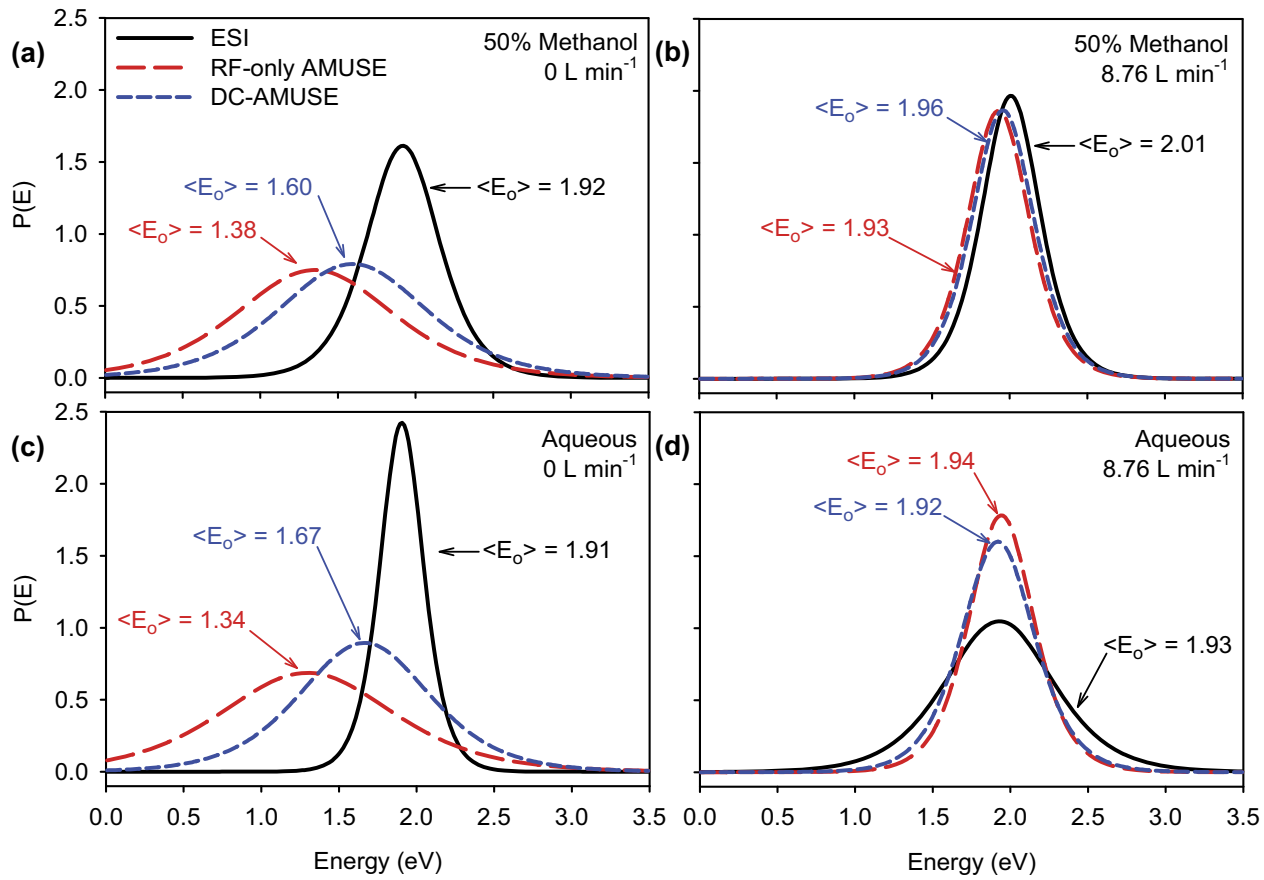

Figure 2. Internal energy distributions for ESI, RF-only AMUSE, and DC-AMUSE ionization of a $5 \mu \mathrm{M}$ mixture prepared in $50 \%$ methanol $(\mathbf{a}, \mathbf{b})$ or water $(\mathbf{c}, \mathbf{d})$ without Venturi focusing $(\mathbf{a}, \mathbf{c})$ or with $8.76 \mathrm{~L} \mathrm{~min}^{-1} \mathrm{~N}_{2}$ flowing through the air amplifier $(\mathbf{b}, \mathbf{d})$. TL $=40 \mathrm{~V}$ and $6 \mathrm{~mm}$ between the air amplifier outlet and MS capillary inlet for all experiments.

sity of the precursor ions. However, an improvement in signal intensity upon using the air amplifier with AMUSE was previously observed for the analysis of less labile compounds [17]. In the absence of $\mathrm{N}_{2}$ flowing through the air amplifier (Figure 2a), RF-only AMUSE ionization was the softest of all techniques, with a mean internal energy of $1.38 \mathrm{eV}$, which is $39 \%$ lower than what was observed for ESI $(1.92 \mathrm{eV})$. The mean internal energy obtained by DC-AMUSE ionization was slightly higher at $1.60 \mathrm{eV}$, possibly as a result of decreased mass transfer arising from condensation buildup, as subsequently discussed in more detail, but was still lower than that for ESI.

Flowing $\mathrm{N}_{2}$ through the air amplifier at $8.76 \mathrm{~L} \mathrm{~min}^{-1}$ resulted in a marked increase in the mean internal energies for AMUSE ionization, and only a 4\% increase for ESI. The mean internal energy for RF-only AMUSE was only marginally lower than that of DC-AMUSE and Venturi-assisted ESI: 1.93, 1.96, and $2.01 \mathrm{eV}$, respectively. The slight increase in the mean internal energy observed for ESI when utilizing Venturi focusing indicates that, under optimum focusing conditions, the use of an air amplifier does not have a detrimental effect on the internal energy deposition. The increase in internal energy observed for the ionization methods can be attributed to changes in droplet size distribution upon use of the air amplifier. In ESI, droplet size is an important factor in determining internal energy deposition [35]. The decreased droplet size arising from use of the air amplifier can be attributed to the increase in droplet velocity, which causes a reduction of droplet/ ion residence time before entering the mass spectrometer heated inlet and results in an effective decrease in ion subcooling associated with solvent evaporation. Increased frictional droplet heating attributed to hydrodynamic friction within the flowing nitrogen is not expected to be of any significance because nitrogen viscosity is very small and there are no significant velocity gradients along the center axis (parallel to the walls) of the air amplifier. Even if there is any small gas heating, the heat capacity of the liquid is much larger than that of the gas, so no appreciable increase in droplet temperature arising from this effect could be expected. Since droplets are carried by the flowing air because of their low inertia attributed to small size, the relative tangential droplet-to-nitrogen velocity is zero. In other words, no slip is expected because droplet and flowing nitrogen gas are moving at the same velocity, resulting in minimal frictional heating.

The ESI droplet size distribution produced in this configuration is shown in Figure S-3a and b. Without the use of Venturi focusing, the mean droplet diameter was $46.0 \mu \mathrm{m}$ with an FWHM value of $16.3 \mu \mathrm{m}$ for ESI (Figure S-3a), which is indicative of considerable droplet coalescence during the transit from the ESI capillary to the MS inlet. Close examination of the droplet size distribution obtained by ESI shows the additional presence of a low-abundance droplet population with a wide diameter distribution ranging from 5 to $20 \mu \mathrm{m}$. The presence of multiple droplet size distributions when the air amplifier is off is not unexpected and can be attributed to the long residence time of droplets, 
which allows for some droplets to emerge individually, whereas others coalesce and exit as larger droplets. The distribution obtained when $\mathrm{N}_{2}$ was allowed to flow through the air amplifier (Figure S-3b) shows a more uniform droplet population with a significantly lower mean diameter and FWHM, 16.1 and $3.5 \mu \mathrm{m}$, respectively, than that obtained without Venturi-assisted droplet entrainment. Because the probability of generating ions via desolvation of the more abundant largediameter population is low, likely the mass spectrometric signal observed during the non-Venturi focusing experiment is attributed mostly to the low abundant small-diameter droplet population mentioned earlier. This would explain the similarities observed for ESI internal energy distributions between Figure $2 a$ and $2 b$ because the spectrometer is sampling from droplet populations having similar diameters.

The mean diameter and FWHM of droplets produced by RF-AMUSE (Figure S-3c) without Venturiassisted transmission were 13.4 and $2.8 \mu \mathrm{m}$, respectively, with a tail reaching values as high as $20 \mu \mathrm{m}$. Since upon ejection AMUSE-generated droplets have a uniform size of $5-7 \mu \mathrm{m}$ (defined by the nozzle aperture) [31], this increase in the mean diameter suggests droplet coalescence during transport to the MS inlet capillary. These larger droplets could be responsible for the lower mean internal energy distributions observed in Figure 2a. The mean droplet diameter and FWHM decreased to 12.7 and $1.8 \mu \mathrm{m}$ when $8.76 \mathrm{~L} \mathrm{~min}^{-1} \mathrm{~N}_{2}$ was allowed to flow through the air amplifier (Figure S-3d) with a concomitant increase in the mean internal energy distribution (Figure $2 b$ ). The intensity of scattered light increased for ESI upon use of the air amplifier (Figure S-3a and b, lower panels), following the creation of a spatially concentrated population of smaller droplets. The scattering intensity correlates with the total number of scatterers/droplets per unit volume and relative size of the droplets compared to the wavelength of light. For RF-only AMUSE, the intensity of light scattered by the spray (Figure S-3c and $\mathrm{d}$, lower panels) was reduced when $\mathrm{N}_{2}$ was allowed to flow through the air amplifier, indicating that desolvation is enhanced by the air amplifier.

To investigate the behavior of Venturi-assisted AMUSE and ESI with solvent systems that are fully compatible with biological species, the previous experiments were repeated using pure water solutions. The results are shown in Figure $2 c$ and $d$, respectively, and corresponding mass spectra are shown in Figure S-4. The analyte signal intensities in the ESI mass spectra (Figure S-4a and b) are one order of magnitude lower for the aqueous samples than for the methanol-containing samples (Figure S-2a and b), which is likely the result of insufficient desolvation, whereas that for the AMUSE mass spectra (Figure S-4c-f) are approximately one order of magnitude higher, which can be attributed to the more effective pressure-based, rather than Taylor-cone-based mechanism of liquid atomization. Comparison of the in- ternal energy distributions shown in Figure 2a versus $2 \mathrm{c}$ and Figure $2 \mathrm{~b}$ versus $2 \mathrm{~d}$ shows that the mean internal energies for aqueous ESI, RF-only AMUSE, and DC-AMUSE were within $5 \%$ of the values obtained using a 50\% methanol solution. The slightly higher mean internal energy observed for DCAMUSE, relative to that for RF-only AMUSE, when operating in non-Venturi mode (Figure 2a and c), can be attributed to decreased mass transfer caused by the buildup of condensation on the external surface of the ejector nozzle array, possibly resulting from electrowetting [36]. This led to significant variations in the ejection flow rate as a result of sporadic blockage of ejector orifices and thus experiments using this approach were not further pursued. Hydrophobic surface functionalization to prevent this effect is now being investigated.

The most salient difference upon changing the solvent system was the $0.5-\mathrm{eV}$ increase in FWHM of the $\mathrm{P}(\mathrm{E})$ curve for Venturi-assisted ESI (Figure $2 \mathrm{~b}$ versus $2 \mathrm{~d}$ ). The explanation of this observation is not straightforward. This is possibly caused either by a decreased evaporative droplet cooling rate [37] or by variations in the droplet size distribution upon changes in solvent composition. There has been only one report on the influence of solvent composition on the internal energy distribution of electrospray droplets that has shown a shift to higher mean energies and wider $\mathrm{P}(\mathrm{E})$ distributions when less volatile solvents with lower vapor pressures are used [33]. However, this is not an obvious result of general value because, although less volatile solvents do indeed evaporate at the lower rates, their latent heat of vaporization is generally much higher and thus capable of inducing more significant subcooling. Droplet size distributions produced for the aqueous solvent by ESI (Figure S-5a and b) show bimodal behavior with two distinct droplet populations. The smaller-size population showed a mean droplet diameter and FWHM of 12.9 and $1.8 \mu \mathrm{m}$, respectively (Figure S-5b), whereas the more abundant population overlaps with the distribution measured without Venturi assistance (Figure S-5a). The presence of these multiple droplet size populations possibly explains the broader internal energy distribution observed for ESI (Figure 2d). The lower desolvation efficiency observed for aqueous ESI is also reflected by the increase in scattered light intensity (Figure S-3b versus Figure S-5b, lower panels), indicative of the presence of a higher volume concentration of droplets. The droplet size distributions and scattered light intensities obtained for RF-only AMUSE ionization of an aqueous solution (Figure S-5c and d) were found to be similar to those obtained for the methanolic solution. The focus of all further experiments was on measuring the effect of the API capillary temperature and nitrogen flow rate on the internal energy landscapes of ESI and RF-only AMUSE. 


\section{Combined Influence of API Capillary Temperature and Air Amplifier Nitrogen Flow Rate on the Internal Energy Deposition}

Recent experiments by Dixon et al. [28] indicated that the use of an air amplifier in combination with ESI provides both a focusing effect and improved droplet desolvation. Varying the air amplifier gas flow rate provides control over both the desolvation efficiency and the aerodynamic focusing of the droplets, resulting in improved ion transport. These two effects are strongly coupled and are expected to influence both internal energy deposition and $\mathrm{S} / \mathrm{N}$ ratio. Drahos and colleagues have shown that for various instruments, including quadrupole and Fourier transform mass spectrometers operated at various ion source temperatures $\left(25-150^{\circ} \mathrm{C}\right)$ the internal energy distribution of ESI ions can be approximated by Boltzmann distribution with different characteristic temperatures [38]. For capillary temperatures of $50-75^{\circ} \mathrm{C}$, Gabelica et al. [34] showed that $\mathrm{P}(\mathrm{E})$ curves exhibit a low-energy tail, indicating the presence of partially solvated ions reaching the capillary-skimmer region of the mass spectrometer.

These previous reports prompted us to perform additional experiments to determine the combined effect of the API capillary temperature and $\mathrm{N}_{2}$ flow rates on the mean internal energy deposited by Venturiassisted ESI and AMUSE and to explore the internal energy landscapes of these techniques. Figure 3 shows contour plots of the mean internal energies for ions produced by either Venturi-assisted ESI (Figure 3a) or RF-only AMUSE (Figure 3b). The overall shape of these surfaces differs in several aspects. Venturi-assisted ESI is characterized by the presence of a low-internalenergy $(\langle\mathrm{E}\rangle<1.5 \mathrm{eV})$ valley in the regime map, occurring at conditions with capillary temperatures of 230$275^{\circ} \mathrm{C}$ and $\mathrm{N}_{2}$ flow rates between 0 and $7 \mathrm{~L} \mathrm{~min}^{-1}$. A region with mean internal energy $\leq 1.0 \mathrm{eV}$ was present at capillary temperatures up to $250{ }^{\circ} \mathrm{C}$ and $\mathrm{N}_{2}$ flow rates between 2 and $4 \mathrm{~L} \mathrm{~min}^{-1}$. The RF-only AMUSE landscape was characterized by a low-internal-energy $(\langle\mathrm{E}\rangle<$ $1.5 \mathrm{eV}$ ) region present at all capillary temperatures tested with $\mathrm{N}_{2}$ flow rates up to $5 \mathrm{~L} \mathrm{~min}^{-1}$. A region with mean internal energy $\leq 1.0 \mathrm{eV}$ was present at capillary temperatures up to $280{ }^{\circ} \mathrm{C}$ and $\mathrm{N}_{2}$ flow rates up to $2.5 \mathrm{~L}$ $\min ^{-1}$. Ions with a mean internal energy $<1.5 \mathrm{eV}$ were obtained in $42 \%$ of the conditions tested by ESI and in $46 \%$ of conditions tested by RF-only AMUSE. Ions with a mean internal energy $\leq 1.0 \mathrm{eV}$ were obtained in 9 and $13 \%$ of conditions tested by ESI and RF-only AMUSE, respectively. The average internal energy obtained by RF-only AMUSE was 7\% lower than that obtained by ESI under all conditions examined.

The shape of the ESI landscape implies that ions of high internal energy can be produced at either high capillary temperatures or high $\mathrm{N}_{2}$ flow rates (Figure $3 \mathrm{a}$ ). The fact that flow rates between 7 and $10 \mathrm{~L} \mathrm{~min}^{-1}$ also result in higher mean internal energy ions, even for capillary temperatures as low as $250{ }^{\circ} \mathrm{C}$, is inherently
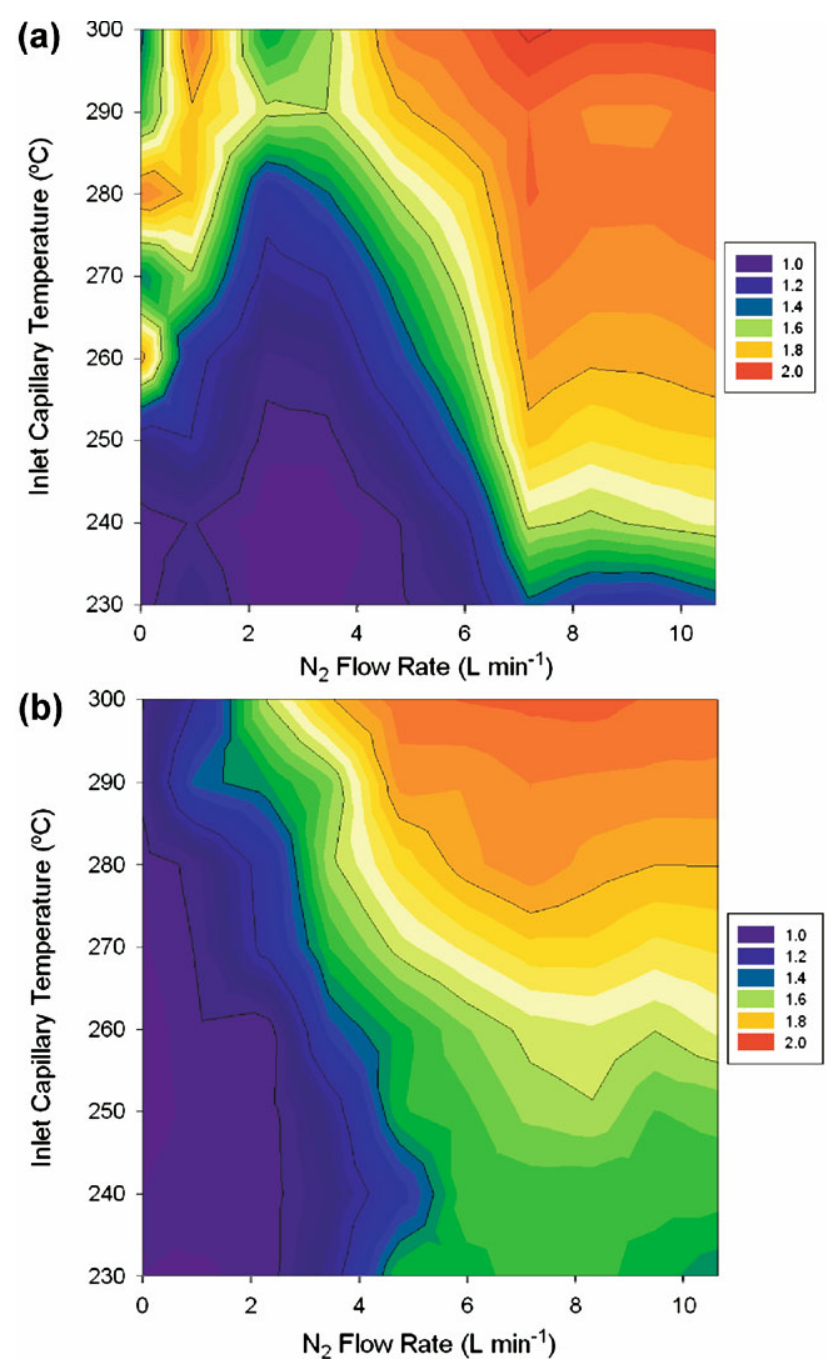

Figure 3. Mean internal energy deposited on ions produced from an aqueous mixture using (a) ESI and (b) RF-only AMUSE ionization at varying capillary temperatures and $\mathrm{N}_{2}$ flow rates from 0 to $10.6 \mathrm{~L} \mathrm{~min}^{-1}$. TL $=30 \mathrm{~V}$ for all experiments.

related to the decrease in residence time as the flow rate of $\mathrm{N}_{2}$ is increased. Shortening the droplet/ion residence time causes a reduction in evaporative cooling, and thus leads to a higher internal energy. The characteristic droplet temperature [38] increases with increasing droplet velocity, resulting in ions being ejected with higher internal energies. Interestingly, at low $\mathrm{N}_{2}$ flow rates and high capillary temperatures, a region of high mean internal energy ESI ions is also observed. In this case, the sensible heat convectively transferred to the droplets during their transit in the heated capillary seems to be sufficient to significantly elevate the ion's internal energy.

The generation of AMUSE ions with high mean internal energy requires conditions combining both high temperatures and high flow rates (Figure $3 b$ ). Because of the different principle used for liquid atomization in AMUSE, the droplets produced are uniform in size [31], even at the extremes of air amplifier flow 
rates (Figure S-5c and d). These droplets are also expected to have lower charge density because of the lower charging potential effectively applied to the liquid reservoir during each RF half-period, resulting in lower coulombic explosion rates. This translates into larger AMUSE droplets surviving longer within the heated capillary. The increase in mean internal energy measured for AMUSE ionization can be attributed to: (1) the increase in capillary temperature, resulting in an equivalent increase in the rate of convective heat transfer to the droplet in combination with (2) the increase in flow rate, resulting in an increase in the convective heat transfer coefficient, which in turn results in increased sensible heat transfer to the droplets. At high flow rates but lower capillary temperatures, the energy transferred at the API inlet results in ions with intermediate mean internal energies.

An investigation of the $\mathrm{S} / \mathrm{N}$ ratio of ions produced by Venturi-assisted ESI and RF-only AMUSE at the upper and lower limits of the investigated capillary temperatures was also performed. The methyl-substituted thermometer compound was selected for this experiment because it has the second lowest critical energy of the compounds tested and can be easily fragmented while still providing a measurable signal for the intact ion $(\mathrm{m} / \mathrm{z}$ 184). The $\mathrm{S} / \mathrm{N}$ ratio for this ion at increasing $\mathrm{N}_{2}$ flow rates and capillary temperatures of $230{ }^{\circ} \mathrm{C}$ (Figure 4a) and $300{ }^{\circ} \mathrm{C}$ (Figure $4 \mathrm{~b}$ ) is presented in Figure 4. Examination of the ESI energy landscape at $230^{\circ} \mathrm{C}$ suggests that the $\mathrm{S} / \mathrm{N}$ ratio of the intact ion should be relatively constant at most of the flow rates tested and should have a maximum value that corresponds to the flow rates that provide the minimum mean internal energy (2-5 $\mathrm{L} \mathrm{min}^{-1}$ ). The observed $\mathrm{S} / \mathrm{N}$ ratio values appear to follow this trend reasonably well, with a maximum $\mathrm{S} / \mathrm{N}$ ratio observed between 4 and $6 \mathrm{~L} \mathrm{~min}^{-1}$. The $\mathrm{S} / \mathrm{N}$ ratio obtained by AMUSE ionization at $230{ }^{\circ} \mathrm{C}$ increases with flow rates up to $2.5 \mathrm{~L} \mathrm{~min}^{-1}$, where it levels off. This correlates well with the AMUSE energy landscape. The observed S/N ratio differences between the two ionization techniques cannot be explained solely based on the ESI and AMUSE internal energy landscapes, but require consideration of the differences in spray divergence and electrochemical processes between these two methods. ESI droplets have a significantly higher charge density than that of AMUSE droplets and thus experience a greater degree of spray divergence. In the case of AMUSE, the increase in signal with $\mathrm{N}_{2}$ flow rate is less dramatic as a result of the lower spray divergence. In addition, the higher potential used in ESI causes higher background intensity than that in AMUSE because of the increased rate of solvent oxidation processes [39] and possibly because of ionization of volatile contaminants present in the laboratory air [40]. As the air amplifier $\mathrm{N}_{2}$ flow rate is increased, the ESI diverging plume is focused toward the mass spectrometer inlet [28], causing an increase not only in the analyte signal intensity but also in the background

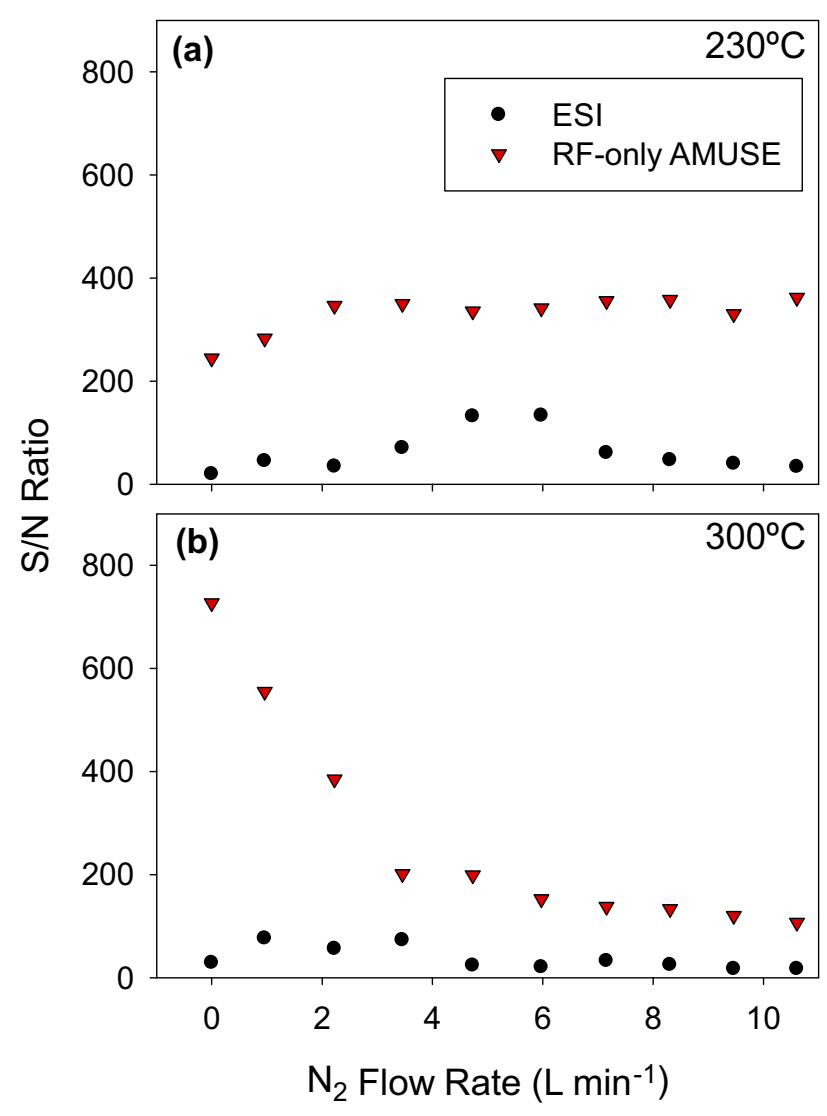

Figure 4. S/N ratio of $\mathrm{m} / \mathrm{z} 184$ peak (precursor ion of methylsubstituted thermometer compound) for Venturi-assisted ESI and RF-only AMUSE ionization of an aqueous mixture at varying $\mathrm{N}_{2}$ flow rates and capillary temperatures of (a) $230^{\circ} \mathrm{C}$ and (b) $300{ }^{\circ} \mathrm{C}$. $\mathrm{TL}=30 \mathrm{~V}$ for all experiments.

intensity, as shown in Figures S-2 and S-4. These effects result in a lower $\mathrm{S} / \mathrm{N}$ ratio for ESI at all assayed flow rates. An examination of the $\mathrm{S} / \mathrm{N}$ ratio changes with air amplifier gas flow rate when the capillary is kept at $300{ }^{\circ} \mathrm{C}$ (Figure $4 \mathrm{~b}$ ) shows that the $\mathrm{S} / \mathrm{N}$ ratio for both ionization methods also correlates well with the trends suggested by the respective internal energy landscapes (Figure 3). The ESI S/N ratio has a local maximum between 1 and $3.5 \mathrm{~L} \mathrm{~min}^{-1}$, which levels off at higher flow rates, whereas the AMUSE S/N ratio decreases sharply as the flow rate is increased up to $3.5 \mathrm{~L} \mathrm{~min}^{-1}$ and exhibits a slower decrease at higher flow rates. The average $\mathrm{S} / \mathrm{N}$ ratio obtained using AMUSE ionization was fivefold higher using a capillary temperature of $230^{\circ} \mathrm{C}$ and eightfold higher than that of ESI at $300^{\circ} \mathrm{C}$.

\section{Conclusions}

We have shown that soft ionization of a series of benzylpyridinium compounds from an aqueous solvent by Venturi-assisted AMUSE and Venturi-assisted ESI ion sources is possible. Our results showed that AMUSE ionization without Venturi assistance is inherently softer than ESI with a mean internal energy that is $39 \%$ lower. AMUSE internal energy deposition was 
significantly modified by increasing the air amplifier gas flow rate, but a less marked effect was observed for ESI. Modulation of the internal energy distribution was accomplished by tuning the $\mathrm{N}_{2}$ gas flow rate in the air amplifier and the API capillary temperature because these parameters most strongly influence desolvation and droplet residence times. Examination of the energy landscapes obtained demonstrated that ions with a mean internal energy $<1.5 \mathrm{eV}$ could be obtained using $46 \%$ of the conditions tested during RF-only AMUSE ionization compared to $42 \%$ of those tested during ESI. A low-internal-energy region $(\langle\mathrm{E}\rangle \leq 1 \mathrm{eV})$ was observed at capillary temperatures up to $250{ }^{\circ} \mathrm{C}$ and $\mathrm{N}_{2}$ flow rates between 2 and $4 \mathrm{~L} \mathrm{~min}^{-1}$ for ESI and at capillary temperatures up to $280^{\circ} \mathrm{C}$ and $\mathrm{N}_{2}$ flow rates up to $2.5 \mathrm{~L}$ $\min ^{-1}$ for AMUSE. Finally, it was shown that at all air amplifier gas flow rates tested, the average $\mathrm{S} / \mathrm{N}$ ratio of the intact methyl-substituted thermometer ion by AMUSE was fivefold higher than that of ESI at $230^{\circ} \mathrm{C}$ and eightfold higher at $300{ }^{\circ} \mathrm{C}$ because of the difference in background noise observed between AMUSE and ESI spectra.

\section{Acknowledgments}

FMF gratefully acknowledges initial financial support received from the American Society for Mass Spectrometry as a Research Award, sponsored by Applied BioSystems and MDS Sciex. CYH acknowledges partial support from the Molecular Biophysics Training Program at the Georgia Institute of Technology. We also thank Prof. David Muddiman at North Carolina State University and Glenn A. Harris at the Georgia Institute of Technology for stimulating discussions. This publication was made possible by Grant Number RR021474-01A1 from the National Center for Research Resources (NCRR), a component of the National Institutes of Health (NIH). Its contents are solely the responsibility of the authors and do not necessarily represent the official views of NCRR or NIH.

\section{References}

1. Tanaka, K.; Waki, H.; Ido, Y.; Akita, S.; Yoshida, Y.; Yoshida, T. Protein and Polymer Analyses up to m/z 100000 by Laser Ionization Time-ofFlight Mass Spectrometry. Rapid Commun. Mass Spectrom. 1988, 2, 151-153

2. Karas, M.; Hillenkamp, F. Laser Desorption Ionization of Proteins with Molecular Masses Exceeding 10,000 Daltons. Anal. Chem. 1988, 60, 2299-2301.

3. Dole, M.; Mack, L. L.; Hines, R. L.; Mobley, R. C.; Ferguson, L. D.; Alice, M. B. Molecular Beam of Macroions. J. Chem. Phys. 1968, 49, 2240-2249.

4. Fenn, J. B.; Mann, M.; Meng, C. K.; Wong, S. F.; Whitehouse, C. M. Electrospray Ionization for Mass Spectrometry of Large Biomolecules. Science 1989, 246, 64-71.

5. Laiko, V. V.; Baldwin, M. A.; Burlingame, A. L. Atmospheric Pressure Matrix-Assisted Laser Desorption/Ionization Mass Spectrometry. Anal. Chem. 2000, 72, 652-657.

6. Hirabayashi, A.; Sakairi, M.; Koizumi, H. Sonic Spray Mass Spectrometry. Anal. Chem. 1995, 67, 2878-2882.

7. Takats, Z.; Wiseman, J. M.; Gologan, B.; Cooks, R. G. Electrosonic Spray Ionization. A Gentle Technique for Generating Folded Proteins and Protein Complexes in the Gas Phase and for Studying Ion-Molecule Reactions at Atmospheric Pressure. Anal. Chem. 2004, 76, 4050-4058.

8. Sakamoto, S.; Fujita, M.; Kim, K.; Yamaguchi, K. Characterization of Self-Assembling Nano-Sized Structures by Means of Coldspray Ionization Mass Spectrometry. Tetrahedron 2000, 56, 955-964.

9. Takats, Z.; Nanita, S. C.; Cooks, R. G.; Schlosser, G.; Vekey, K. Amino Acid Clusters Formed by Sonic Spray Ionization. Anal. Chem. 2003, 75, 1514-1523.

10. Konig, S.; Kollas, O.; Dreisewerd, K. Generation of Highly Charged Peptide and Protein Ions by Atmospheric Pressure Matrix-Assisted
Infrared Laser Desorption/Ionization Ion Trap Mass Spectrometry. Anal. Chem. 2007, 79, 5484-5488.

11. Hirabayashi, A.; Hirabayashi, Y.; Sakairi, M.; Koizumi, H. MultiplyCharged Ion Formation by Sonic Spray. Rapid Commun. Mass Spectrom. 1996, 10, 1703-1705.

12. Nishimura, S.; Nagahori, N.; Takaya, K.; Tachibana, Y.; Miura, N. Monde, K. Direct Observation of Sugar-Protein, Sugar-Sugar, and Sugar-Water Complexes by Cold-Spray Ionization Time-of-Flight Mass Spectrometry. Angew. Chem. Int. Ed. 2005, 44, 571-575.

13. Sakamoto, S.; Yamaguchi, K. Low tm DNA Duplexes Observed by Cold-Spray Ionization Mass Spectrometry. Tetrahedron. Lett. 2003, 44, 3341-3344.

14. Fedorov, A. G.; Degertekin, F. L. Electrospray Systems and Methods. U.S. Patent 7208 727, 2007.

15. Aderogba, S.; Meacham, J. M.; Degertekin, F. L.; Fedorov, A. G.; Fernández F. M. Nanoelectrospray Ion Generation for High-Throughput Mass Spectrometry Using a Micromachined Ultrasonic Ejector Array. Appl. Phys. Lett. 2005, 86, 203110/203111-203110/203113.

16. Meacham, J.; Varady, M.; Degertekin, F.; Fedorov, A. Droplet Formation and Ejection from a Micromachined Ultrasonic Droplet Generator: Visualization and Scaling. Phys. Fluids 2005, 17, 100605/100601-100605/ 100608.

17. Hampton, C. Y.; Forbes, T. P.; Varady, M. J.; Meacham, J. M.; Fedorov, A. G.; Degertekin, F. L.; Fernandez, F. M. Analytical Performance of a Venturi-Assisted Array of Micromachined Ultrasonic Electrosprays Coupled to Ion Trap Mass Spectrometry for the Analysis of Peptides and Proteins. Anal. Chem. 2007, 79, 8154-8161.

18. Chowdhury, S. K.; Chait, B. T. Method for the Electrospray Ionization of Highly Conductive Aqueous Solutions. Anal. Chem. 1991, 63, 1660-1664.

19. Forbes, T. P.; Degertekin, F. L.; Fedorov, A. G. Multiplexed Operation of a Micromachined Ultrasonic Droplet Ejector Array. Rev. Sci. Instrum. 2007, 78, 104101

20. Zook, D. R.; Bruins, A. P. On Cluster Ions, Ion Transmission, and Linear Dynamic Range Limitations in Electrospray (Ionspray) Mass Spectrometry. Int. J. Mass Spectrom. Ion Processes. 1997, 162, 129-147.

21. Zhou, L.; Yue, B.; Dearden, D. V.; Lee, E. D.; Rockwood, A. L.; Lee, M. L. Incorporation of a Venturi Device in Electrospray Ionization. Anal. Chem. 2003, 75, 5978-5983.

22. Yang, P.; Cooks, R. G.; Ouyang, Z.; Hawkridge, A. M.; Muddiman, D. C. Gentle Protein Ionization Assisted by High-Velocity Gas Flow. Anal. Chem. 2005, 77, 6174-6183.

23. Prior, D. C.; Price, J.; Bruce, J. E. Sample Inlet Tube for Ion Source. U.S. Patent 6455 846, 2002.

24. Wu, S.; Zhang, K.; Kaiser, N. K.; Bruce, J. E. Incorporation of a Flared Inlet Capillary Tube on a Fourier Transform Ion Cyclotron Resonance Mass Spectrometer. J. Am. Soc. Mass Spectrom. 2006, 17, 772-779.

25. Dixon, R. B.; Muddiman, D. C. Quantitative Comparison of a Flared and a Standard Heated Metal Capillary Inlet with a Voltage-Assisted Air Amplifier on an Electrospray Ionization Linear Ion trap Mass Spectrometer. Rapid Commun. Mass Spectrom. 2007, 21, 3207-3212.

26. Shaffer, S. A.; Tang, K. Q.; Anderson, G. A.; Prior, D. C.; Udseth, H. R.; Smith, R. D. A Novel Ion Funnel for Focusing Ions at Elevated Pressure Using Electrospray Ionization Mass Spectrometry. Rapid Commun. Mass Spectrom. 1997, 11, 1813-1817.

27. Him, T.; Tolmachev, A. V.; Harkewicz, R.; Prior, D. C.; Anderson, G.; Udseth, H. R.; Smith, R. D.; Bailey, T. H.; Rakov, S.; Futrell, J. H. Design and Implementation of a New Electrodynamic Ion Funnel. Anal. Chem. 2000, 72, 2247-2255.

28. Dixon, R. B.; Muddiman, D. C.; Hawkridge, A. M.; Fedorov, A. G. Probing the Mechanisms of an Air Amplifier Using a LTQ-FT-ICR-MS and Fluorescence Spectroscopy. J. Am. Soc. Mass Spectrom. 2007, 18, 1909-1913.

29. Hawkridge, A. M.; Zhou, L.; Lee, M. L.; Muddiman, D. C. Analytical Performance of a Venturi Device Integrated into an Electrospray Ionization Fourier Transform Ion Cyclotron Resonance Mass Spectrometer for Analysis of Nucleic Acids. Anal. Chem. 2004, 76, 4118-4122.

30. Katritzky, A. R.; Watson, C. H.; Dega-Szafran, Z.; Eyler, J. R. CollisionallyActivated Dissociation of n-Alkylpyridinium Cations to Pyridine and Alkyl Cations in the Gas Phase. J. Am. Chem. Soc. 1990, 112, 2471-2478.

31. Meacham, J. M. A Micromachined Ultrasonic Droplet Generator: Design, Fabrication, Visualization, and Modeling (Ph.D. Thesis), George W. Woodruff School of Mechanical Engineering, Georgia Institute of Technology: Atlanta, 2006.

32. Kenttamaa, H. I.; Cooks, R. G. Internal Energy-Distributions Acquired through Collisional Activation at Low and High-Energies. Int. J. Mass Spectrom. Ion Process. 1985, 64, 79-83.

33. Collette, C.; De Pauw, E. Calibration of the Internal Energy Distribution of Ions Produced by Electrospray. Rapid Commun. Mass Spectrom. 1998, $12,165-170$.

34. Gabelica, V.; De Pauw, E. D.; Karas, M. Influence of the Capillary Temperature and the Source Pressure on the Internal Energy Distribution of Electrosprayed Ions. Int. J. Mass Spectrom. 2004, 231, 189-195.

35. Nefliu, M.; Smith, J. N.; Venter, A.; Cooks, R. G. Internal Energy Distributions in Desorption Electrospray Ionization (DESI). J. Am. Soc. Mass Spectrom. 2008, 19, 420-427.

36. Verheijen, H. J. J.; Prins, M. W. J. Reversibly Electrowetting and Trapping of Charge: Model and Experiments. Langmuir 1999, 15, 6616-6620. 
37. Fisenko, S. P.; Wang, W. N.; Lenggoro, I. W.; Okyuama, K. Evaporative Cooling of Micron-Sized Droplets in a Low-Pressure Aerosol Reactor. Chem. Eng. Sci. 2006, 61, 6029-6034.

38. Drahos, L.; Heeren, R. M. A.; Collette, C.; De Pauw, E.; Vekey, K. Thermal Energy Distribution Observed in Electrospray Ionization. J. Mass Spectrom. 1999, 34, 1373-1379.
39. Zhou, S. L.; Prebyl, B. S.; Cook, K. D. Profiling pH Changes in the Electrospray Plume. Anal. Chem. 2002, 74, 4885-4888.

40. Manier, M. L.; Cornett, D. S.; Hachey, D. L.; Caprioli, R. M. Identification of Dimethyidioctadecylammonium Ion $(\mathrm{m} / \mathrm{z} 550.6)$ and Related Species $(m / z$ 522.6, 494.6) as a Source of Contamination in Mass Spectrometry. J. Am. Soc. Mass Spectrom. 2008, 19, 666-670. 